\title{
ON ASYMPTOTIC CONJUGATE POINTS
}

\author{
YASUO NASU
}

(Received May 30, 1955)

In a simply connected Riemannian space with non-positive curvature, $\mathbf{E}$. Cartan [1] dealt with a particular class of geodesics called asymptotes. $\mathrm{H}$. Busemann [2] extended the concept of asymptotes to the case of $E$-spaces defined by him. In this note the initial point of an asymptote will be called, if it exists, its asymptotic conjugate point (\$2). We study the set of asymtotic conjugate points to a given ray and show some examples to make clear the circumstances.

1. In this paragraph we explain some preliminary concepts.

In a metric spacel) points will always be denoted by small roman letters and the distance between two points $x$ and $y$ will be denoted by $x y$. The axioms for a space $\mathbb{E}$ to be an $E$-space are these:

A. $(5)$ is metric with distance $x y$ not necessarily symmetric.

B. (F is finitely compact, i. e., every bounded subset $X$ contains a sequence of points $\left\{x_{v}\right\}$ which converges to a point $x$ in 3 .

C. $[5$ is convex metric, i.e., for every pair of two distinct points $x$ and $y$ a point $z$ with $x z+z y=x y$ exists.

D. Every point $x$ has a spherical neighborhood $s\left(x, \alpha_{x}\right)=\left\{y \mid y x<\alpha_{x}, x y<\right.$ $\left.\alpha_{x}\right\}\left(\alpha_{x}>0\right)$ such that for any positive number $\varepsilon$ and any two points $a, b \in$ $S\left(x, \alpha_{x}\right)$ there exists positive numbers $\delta_{k}(a, b)(\leqq \varepsilon)(k=1,2)$ for which a point $a_{1}$ with $a_{1} a+a b=a_{1} b$ and $a_{1} a=\delta_{1}$ and another point $b_{1}$ with $a b+b b_{1}=a b_{1}$ and $b b_{1}=\delta_{2}$ exist and are unique.

In an $E$-space, for any two points $x$ and $y$ the axioms $\mathrm{A}, \mathrm{B}$, and $\mathrm{C}$ guarantee the existence of a segment $T(x, y)($ or $T(y, x)$ ) from $x$ to $y$ (or from $y$ to $x$ ) whose length is equal to the distance $x y$ (or $y x$ ). Furthermore the prolongation of an arbitrary segment is locally possible and unique under the axiom D. The whole prolongation of a segment is called an extremal. An extremal $\mathfrak{x}$ has a parametric representation $x(\tau),-\infty<\tau<+\infty$, such that for every $\tau_{0}$ a positive number $\delta\left(\tau_{0}\right)$ exists such that $x\left(\tau_{1}\right) x\left(\tau_{2}\right)=\tau_{2}-\tau_{1}$ for $\tau_{2} \geqq \tau_{1}$ and $\left|\tau_{0}-\tau_{i}\right| \leqq \delta\left(\tau_{c}\right)(i=1,2)$.

An extremal $\mathfrak{x}$ is called a straight line, if its parametric representation $x(\tau),-\infty<\tau<+\infty$, has the property $x\left(\tau_{1}\right) x\left(\tau_{2}\right)=\tau_{2}-\tau_{1}\left(\tau_{2} \geqq \tau_{1}\right)$. A positive ray is a positive half extremal $x(\tau), 0 \leqq \tau<+\infty$, for which $x\left(\tau_{1}\right) x\left(\tau_{2}\right)=\tau_{2}$ $-\tau_{1}\left(\tau_{2} \geqq \tau_{1}\right)$. Similarly negative rays are defined. Our consideration will be

1) In this paper, we use the "metric space" for a set (5) with the following properties (1), (2), (3), (4).

(1) $x y$ is defined for any ordered pair of points $x$ and $y$ in $(5$ and is non-negative.

(2) $x y=0$, if and only if $x=y$.

(3) $x y+y z \geqq x z$ for any three points $x, y$, and $z$.

(4) For any sequence of points $\left\{x_{\nu}\right\}$ and a point $x, x x_{\nu} \rightarrow 0$ if and only if $x_{\nu} x \rightarrow 0$. 
restricted to positive rays and positive half extremals, since the developments are entirely parallel and therefore the term "positive" will be omitted.

In [2], the number $\eta_{\lambda}(x)(\lambda \geqq 2)$ and the term "direction" were introduced. The number $\eta_{\lambda}(x)$ is defined as the least upper bound of those $\beta$ 's for which every segment $T(a, b)(a, b \in S(x, \beta))$ is a cocentral subsegment of a segment of length $\lambda \beta . \eta_{\lambda}(x)$ is positive for an arbitrary point $x$ in $(5$ and an arbitrary positive number $\lambda$ not less than 2. A segment $T$ with $x$ as an initial point is called a direction at $x$, if the length of $T$ is equal to $\eta(x)=\min \left(\eta_{6}(x), 1\right)$.

For two points $x$ and $y \max (x y, y x)$ is denoted by $\sigma(x, y)$. If $D_{1}$ and $D_{2}$ are respectively the initial directions of half extremals $\mathfrak{x}_{1}$ and $\mathfrak{x}_{2}$, then the distance between the two half extremals $\mathfrak{x}_{1}$ and $\mathfrak{x}_{2}$ is defined by

$$
\sigma\left(\mathfrak{x}_{1}, \mathfrak{x}_{2}\right)=\left\{\sigma\left(p_{1}, p_{2}\right)+\sigma\left(q_{1}, q_{2}\right)\right\} / 2,
$$

where $p_{1}, p_{2}$ are respectively the initial points of $D_{1}, D_{2}$ and $q_{1}, q_{2}$ the end points of $D_{1}, D_{2}$. The set of half extremals is not necessarily finitely compact under the metric $(1,1)$ [2].

Let a ray $\{$ ba given, let $x(\tau), 0 \leqq \tau<+\infty$, be its parametric representation, let $\left\{p_{\nu}\right\}$ be a sequence of points which converges to a point $p$, and let $\left\{\tau_{v}\right\}$ be a sequence of positive numbers such that $\tau_{\nu} \rightarrow+\infty$ as $\nu \rightarrow+\infty$. The sequence of segments $\left\{T_{\nu}\right\}\left(T_{\nu}=\left(p_{1}, x\left(\tau_{\nu}\right)\right), \nu=1,2, \ldots \ldots\right)$ contains a subsequence converging in the sense of the metric $(1,1)$ to a ray $r$ with the initial point $p$. $x$ is said a coray from the point $p$ to the ray $r$. The following theorem (1.2) and the theorem below (1.5) are necessary for our later purposes.

(1.2) In an $E$-space 0 , let $\mathfrak{l}$ be a ray, and let $\mathfrak{X}$ be the set of all corays to the ray $l$. The set $\mathfrak{X}$ forms a closed subset of the set of all half extremals.

Let $\left.x_{i}^{\prime} \tau\right), 0 \leqq \tau<+\infty$, be the parametric representation of a ray $\mathfrak{l}$. In [2] the function $\alpha(p, \mathfrak{l})$ is defined as follows:

$$
\alpha(p, \mathfrak{l})=\lim _{\tau \rightarrow+\infty}(p x(\tau)-\tau) .
$$

This limit exists for every point $p$ and every ray $\mathfrak{l}$. By use of this function, the limit sphere through a point $p$ is defined as the set of points $x$ satisfying the following relation:

$$
L(p, \mathfrak{l}): \quad \alpha(p, \mathfrak{l})=\alpha(x, \mathfrak{l}) .
$$

The following theorem is fundamental.

(1.5) In an $E$-space (5, let $\mathfrak{l}$ be a ray. Every half extremal is a coray to , if and only if its parametric representation! $x(\tau), 0 \leqq \tau<+\infty$, satisfies the following condition;

$$
\alpha\left(x\left(\tau_{0}\right), \mathfrak{l}\right)-\alpha\left(x\left(\tau_{1}\right), \mathfrak{l}\right)=\tau_{1}-\tau_{0} \text { for } \tau_{0}, \tau_{1} \geqq 0 .
$$

As can be seen from the definition, the relation $|\alpha(p, \mathfrak{l})-\alpha(q, \mathfrak{l})| \leqq \sigma(p, q)$ holds for any two points $p$ and $q$. Therefore if $\tau_{1}>\tau_{0}$, then $x\left(\tau_{1}\right)$ is a foot ${ }^{3)}$

2) H. Busamann [2] proved that the convergence of a subsequence of $\left\{T_{\nu}\right\}$ is equivalent to the convergence in the sense of the closed limit introduced by Hausdorff [2], [4].

3) Let a point $p$ and a set $E$ be given. A point $f$ of $E$ is said to be a foot of $p$ on $E$, if $p f \leqq p x$ holds for every point $x$ of $E$. 
of $x\left(\tau_{0}\right)$ on $L\left(x\left(\tau_{1}\right), \mathfrak{l}\right)$. Hence every coray is perpendicular to limit spheres which intersect it. From the theorem (1.5) and the definition of corays, there does not necessarily exist another coray containing a coray as a subray, but every subray of a coray is also a coray. An asymptote to a ray is defined as the union of all corays containing a coray as a subray.

2. An asimptote has an initial point, unless it is a straight line. The initial point of an asymptote $\mathfrak{U}$ to a ray $\mathfrak{l}$ is said the asymptotic conjugate point of $\mathfrak{i}$ to $\mathfrak{l}$. Hereafter the set of asymptotic conjugate points to a ray $\mathfrak{l}$ will be denoted by $K(\mathfrak{l})$. From the definition of asymptotes we see that any asymptote is also a coray whose initial point is an asymptotic conjugate point.

Then the following (2.1) is to be noticed.

(2.1) Let $\mathfrak{i l}$ be an asymptote to a ray $\mathfrak{l}$ and $a$ be its initial point. A coray from every point of $\mathfrak{A}-a$ to $\mathfrak{l}$ is a subray of $\mathfrak{A}$.

To make clear the above explanation, we show the following example.

EXAMPLE $1^{\circ}$. In a 3-dimensional euclidean space referred to the rectangular coordinate system $(x, y, z)$, cut off from the $x y$-plane the circular disk whose center is $(1,1,0)$ and diameter $1 / 2$, and join the half cylinder :

$$
(x-1)^{2}+(y-1)^{2}=1 / 4, \quad z \geqq 0 .
$$

Then we have a surface $S^{\prime}$ instead of the $x y$-plane. To get a smooth surface we make use of an arc of the algebraic curve $C$ which is expressed by a rectangular coordinate system $(\xi, \eta)$ as follows:

$\alpha(\xi-1 / 4)^{4}+\alpha(\eta-1 / 4)^{4}+2 \beta \xi^{2} \eta^{2}-\alpha / 4^{4}=0(\alpha>0, \beta>0)$.

Thelcurve $C$ is in contact of third order with $\xi, \eta$-axes at $(1 / 4,0)$ and $(0,1 / 4)$.

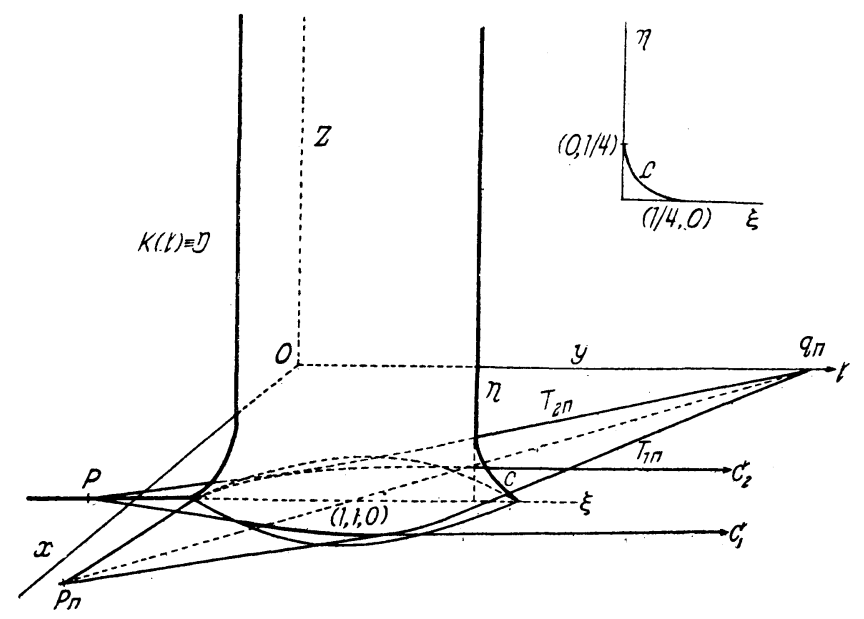

As shown in the figures, by use of $C$ we smooth every section of $S^{\prime}$ by a plane through the axis of the half cylinder. Thus we have a smooth surface $S$.

On this surface $S$, let $\mathfrak{l}$ be the half straight line: 


$$
x=0,0 \leqq y<+\infty, z=0,
$$

and $\left\{p_{n}\right\}\left(p_{n}=\left(x_{n}, y_{n}, z_{13}\right), x_{n}>1,-\infty<y_{n} \leqq 1 / 2, z_{n} \geqq 0\right)$ be a sequence of points with the limit point $p$ on the curve $y$ which is the intersection of $S$ and the half plane:

$$
x=1,-\infty<y \leqq 1 .
$$

Further, let the plane through $p_{n}$ and the axis of the half cylinder intersect $y$-axis at a point $q_{n}$. Then $\boldsymbol{q}_{n}$ is given by $\left(0,\left(x_{n}-y_{n}\right) /\left(x_{n}-1\right), 0\right)$ and $\lim _{n \rightarrow \infty}$ $\left(x_{n}-y_{n}\right) /\left(x_{n}-1\right)=+\infty$. Obviously there exist two segments from $p_{n}$ to $q_{n}$ which are not homotopic. We suppose to denote them by $T_{1 n}$ and $T_{2 n}$ such that the closed curves $T(p, p)+T_{11}+T\left(q_{1}, q_{n}\right)+T_{1 n}^{-1}+T\left(p_{n}, p\right)$ and $T\left(p, p_{1}\right)+T_{21}$ $+T\left(q_{1}, q_{n}\right)+T_{2 n}^{-1}+T\left(p_{n}, p\right)$ are homotopic to zero. By use of the sequences $\left\{T_{1 n}\right\}$ and $\left\{T_{2 n}\right\}$ we can easily see that there exist at least two corays $\mathfrak{x}_{1}$

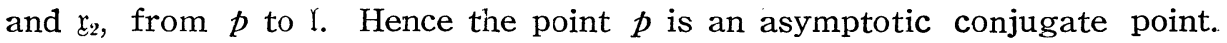
From the above we also see that the curve $\mathfrak{y}$ coincides with the set $K(\mathfrak{l})$.

3. At first we shall prove the following lemma.

(3.1) In an E-space (5, let a ray $\mathfrak{l}$ be given. For a point $p$ of the set $K(\mathfrak{l})$. and a positive number $d$, there exists a positive number $\varepsilon$ such that all corays whose initial points belong to $-S(p, d)$ are disjoint from $S(p, \varepsilon)$.

PROOF. If this were not so, then we should have a sequence of points $\left\{p_{n}\right\}$ converging to the point $p$ such that each point $p_{n}$ lies on a coray $\mathfrak{x}_{n}$ with the initial point $q_{n}$ in $\varepsilon-S(p, d)$. For a positive number $\varepsilon$ there exists a positive integer $N$ such that $\sigma\left(p_{n}, p\right)<\varepsilon$ for every $n \geqq N$. In this case we can take $\varepsilon$ such that $d / 4>\varepsilon>0$. We then have $q_{n} p_{n} \geqq q_{n} p-p_{n} p \geqq d-\varepsilon$ $>3 d / 4>0$. Hence we can take a point $q_{n}^{\prime}$ on $\mathfrak{x}_{n}$ such that $0<\boldsymbol{q}_{n}^{\prime} \boldsymbol{p}_{n}=\delta<d / 4$, $p_{n} q_{n}^{\prime}<d / 4$ and $q_{n}^{\prime} \bar{\in} K(\mathfrak{l})$ for every $n \geqq N$.

Let $\mathfrak{x}_{n}^{\prime}$ be a coray with the initial point $q_{n}^{\prime}(n=N, N+1, \ldots)$. $\mathfrak{x}_{n}$ is a subray of $\mathfrak{x}_{n}(n=N, N+1, \ldots)$. By (1.2) we can see that the sequence $\left\{\mathfrak{x}_{n}^{\prime}\right\}$ contains a subsequence $\left\{\mathfrak{x}_{n_{k}}^{\prime}\right\}$ converging to a coray $\mathfrak{x}^{\prime}$. Then the sequence $\left\{q_{n_{k}}^{\prime}\right\}$ converges to a point $\boldsymbol{q}^{\prime}$. $\mathfrak{x}^{\prime}$ contains $p$ and its initial point $\boldsymbol{q}^{\prime}$ does not coincide with $p$, since $q^{\prime} p=\delta>0$. This contradicts $p \in K(\mathfrak{l})$. Thus the lemma is proved.

(3.2) TheOREM. In an E-space $(\mathfrak{E}$, if the set $K(\mathfrak{l})$ of asymptotic conjugate points to a ray $\mathfrak{l}$ contains an isolated point, then the set $K(\mathfrak{l})$ is composed of only one point $p$ and all asymptotes, with the initial point $p$ simply cover the whole space 3 .

ProOF. Under the assumption of the theorem we can take a positive number $\delta$ such that $(S(p, \delta)-p) \cap K(\mathfrak{l})=\phi$. By (2.1) there exists a positive number $\varepsilon(<\delta)$ such that all corays with initial points in $E-S(p, \delta)$ are disjoint from $S(p, \varepsilon)$.

In the same way as in the proof of (2.1) we can prove that a positive number $\varepsilon^{\prime}$ exists such that every asymptote which is straight line is disjoint from $S\left(p, \varepsilon^{\prime}\right)$. Put $\varepsilon^{\prime \prime}=\min \left(\varepsilon, \varepsilon^{\prime}\right)$. Then we see that every asymptote con- 
taining a point $S\left(p, \varepsilon^{\prime \prime}\right)$ has $p$ as its initial point. From this it follows that $S\left(\not, \varepsilon^{\prime \prime}\right)$ is simply covered by asympototes with the initial point $p$.

If $q$ is a point of $(\mathbb{E}-S(p, \delta)) \cap K(\mathfrak{l})$, then a half extremal issuing from $p$ and passing through $q$ is a coray to the ray $l$, which contradicts $q \in$ (E) $-S(p, \delta)) \cap K(\mathfrak{l})$. This fact yields $(\mathfrak{5}-S(p, \delta)) \cap K(\mathfrak{l})=\phi$. Similarly we can easily prove that, if there were an asymptote which is a straight line, then we should arrive at a contradiction. Hence the theorem is proved.

(3.3) Under the assumption of (3.2) every sphere with the center coincides with a limit sphere.

PROOF. Let $q$ be a point of a sphere $K(p, \rho)(=\{x \mid p x=\rho\})$. The asymptote through the point $q$ is perpendicular to the limit sphere through $q$. Hence we have by (1.5)

$$
\alpha(p, \mathfrak{l})-\alpha(q, \mathfrak{l})=\rho(=p q) .
$$

Since $q$ is an arbitrary point of $K(p, \rho)$, we have, by the above relation,

Therefore

$$
\alpha(\boldsymbol{q}, \mathfrak{l})=\alpha(x, \mathfrak{l}) \text { for every point } x \in K(p, \rho) .
$$

$$
K(p, \rho) \subset L(q, \mathfrak{l}) \text {. }
$$

Conversely, let $x$ be a point of $L(q, \mathfrak{l})$. The point $p$ has a foot $x$ on $L(q, \mathfrak{l})$, since the asymptote through $x$ is perpendicular to $L(q, \mathfrak{l})$ at $x$. From this it follows that

$$
K(p, \rho) \supset L(q, \mathfrak{l})
$$

Thus the theorem is proved.

In the theorem (3.3), if $q$ is a point on $\mathfrak{l}$, then the asymptote $\mathfrak{A}$ through $q$ contains the ray $\mathfrak{l}$. Hence the ray $\mathfrak{l}$ is a subray of $\mathfrak{I}$, and the limit sphere $L(p, \mathfrak{l})$ is composed of only one point $p$.

EXAMPLE $2^{\circ}$. Let $(x, y, z)$ be a rectangular coordinate system of a 2 dimensional euclidean space, and consider the surface defined by $x^{2}+y^{2}=1$ for $z \geqq 0$ and $x^{2}+y^{2}+z^{2}=1$ for $z<0$ and a generating line on $x^{2}+y^{2}=1$, $z \geqq 0$ as a given ray $\mathfrak{l}$. It can easily be seen that the set $K(\mathfrak{l})$ is composed of only one point $(0,0,-1)$

(3.4) In an $E$-space (5, the set $K(l)$ to a ray 1 is not necessarily closed.

The above fact shows us that the limit point of a Cauchy sequence of asymptotic conjugate points does not necessarily belong to the set $K(\mathfrak{l})$. This fact will be shown by the following example.

EXAMPLE $3^{\circ}$. Let $(x, y, z)$ be a rectangular coordinate system of a 3 dimensional euclidean space. Consider the sequence $\left\{p_{n}\right\}$ where $p_{n}$ is given by $((2 n+1) / 2 n(n+1), n, 0)$, and replace the circular disk whose center is $p_{n}$ and diameter $1 / 4 n(n+1)$ by the half cylinder $Z_{n}$ :

$$
(x-(2 n+1) / 2 n(n+1))^{2}+(y-n)^{2}=1 / 16 n^{2}(n+1)^{2}, \quad z \geqq 0(n=1,2, \ldots) .
$$

In the same way as in the example $1^{\circ}$ we smooth the joint part of $Z_{n}$ and the $x y$-plane by use of the algebraic curve $C_{n}$ :

$$
\begin{gathered}
\alpha\left(\xi-\gamma_{n}\right)^{4}+\alpha\left(\eta-\gamma_{n}\right)^{4}-2 \beta \xi^{2} \eta^{2}-\alpha \gamma_{n}^{4}=0\left(\alpha>0, \beta>0, \gamma_{n}=1 / 8 n(n+1)\right) \\
(n=1,2, \ldots) .
\end{gathered}
$$


Let the surface thus obtained be $S$. On the surface $S$, let $\mathfrak{l}$ be the half straight line :

$$
x=1,0 \leqq y<+\infty, z=0 .
$$

Then the system of half straight lines:

$$
x=(2 n+1) / 2 n(n+1), \quad-\infty<y \leqq n-3 / 8 n(n+1)(n=1,2, \ldots)
$$

is contained in the set $K(\mathfrak{l})$. Hence the sequence of points $\left\{\boldsymbol{q}_{n}\right\} \quad\left(\boldsymbol{q}_{n}=((2 n+\right.$ $1) / 2 n(n+1), \lambda, 0), 0<\lambda \leqq 13 / 16)$ is contained in the set $K(\mathfrak{l})$ and converges to the point $(0, \lambda, 0)$. But $y$-axis $(x=0,-\infty<y<+\infty, z=0)$ is an asymptote to the ray $\Upsilon$. Hence $(0, \lambda, 0) \in K(\mathfrak{l})$. This proves (3.4).

In the above construction of the surface $S$, we can get a differentiable surface of any order by use of suitable algebraic curves. Hence we can get a Riemannian space of any class in such a way that the set $K(\mathfrak{l})$ for a ray $\mathfrak{l}$ is not closed. The se $\tau$ of asymptotic conjugate points in Example $2^{\circ}$ is closed but in Example $3^{\circ}$ is not closed.

REMARK. In order to get a 2-dimensional E-space, it is sufficient that the above smoothing is of class $C^{\prime 2}$. This fact easily be seen from the result obtained by P. Hartmann and A. Wintner [5].

4. In this paragraph we shall consider the case where the set of asymptotic conjugate points to a ray $\mathfrak{l}$ is closed.

The set $K(\mathfrak{l})$ is closed, if an arbitrary point $p(\bar{\epsilon} K(\mathfrak{l}))$ has a neighborhood simply covered by a system of asymptotes whose initial point do not belong to this neighborhood.

At first we shall prove the following

(4.1) THEOREM. In an E-space (5, let a ray $\mathfrak{l}$ be given and a ray $x$ be a coray from a point $p$ to $l$. If the set $K(l)$ is closed, then for an arbitrary positive number $\rho$ every point $q(\neq p)$ on $\mathfrak{x}$ has a neighborhood simply covered by $a$ system of asymptotes containing a point in $S(p, \rho)$.

Proof. If this were not so, we should have a sequence of points $\left\{\boldsymbol{q}_{n}\right\}$ ( $q_{n} \in K(\mathfrak{l}), \quad n=1,2, \ldots$ ) converging to the point $q$ such that the asymptote $\mathfrak{H}_{n b}$ through each point $q_{n b}$ is disjoint from $S(p, \rho)$, sincesthe set $K(\mathfrak{l})$ is closed and $q \bar{\epsilon} K(\mathfrak{l})$.

Let $\mathfrak{A}$ be the asymptote through $q$ and $\mathfrak{x}_{n}$ be a coray from each $q_{n}$ to $\mathfrak{l}$. The $\mathfrak{x}_{n}$ is a subray of $\mathfrak{A}_{n}$, and the sequence of corays $\left\{\mathfrak{x}_{n}\right\}$ converges to the subray $\mathfrak{x}$ of $\mathfrak{A}$, since $q \in \bar{E} K(\mathfrak{l})$. Hence the sequence of asymptotes $\left\{\mathfrak{P}_{n}\right\}$ converges to the asymptote $\mathfrak{H}$. From this a positive integer $N$ exists such that $S(p, \rho) \cap \mathfrak{A}_{n} \neq \phi$, for every $n \geqq N$, which contradicts the assumption. Thus the theorem is proved.

The converse evidently follows from the fact mentioned at the beginning of this paragraph.

H. Busemann proved the following 
(4.2) If an E-space (5 is a straight line space, then every asymptote to a ray $\mathfrak{l}$ is a straight line.

From the above theorem, we see that in a straight line space the set $K(\mathfrak{l})$ to a ray $\mathfrak{l}$ is always empty, in other words, there exists only one coray from every point to a ray $l$.

In an E-space 5 , if every subset homeomorphic to an open subset is open in $\mathbb{E}$, then $\mathbb{E}$ is said a space with the property of domain invariance [3]. Then we have the following

(4.3) Theorem. In an E-space with the property of domain invariance, if there exists only one coray from every point to a ray $\Upsilon$, then the set $K(\mathfrak{l})$ is closed.

Proof. Let $\left\{p_{n}\right\}$ be a Cauchy sequence in the set $K(\mathfrak{l})$. Then $\left\{p_{n}\right\}$ converges to a point $p$. It is sufficient to prove $p \in K(\mathfrak{l})$. If this were not so, then the coray from $p$ to $\mathfrak{l}$ should be a proper subray of an asymptote $\mathfrak{A}$. We take on $\mathfrak{A}$ a point $q$ before $p$. Let $S(q, \alpha)$ be a spherical neighborhood such that $q p>\alpha$. If we take a point $x^{\prime}$ on the coray from a point $x$ of $S(q, \alpha)$ such that $x x^{\prime}=q p$, then this correspondence is one-to-one. Furthermore this correspondence is bicontinuous. Therefore the set $S^{\prime}(\ni p)$ composed of such points $x^{\prime}$ is homeomorphic to $S(q, \alpha)$. By virtue of the assumption of the theorem $S^{\prime}$ is open in $\left(5\right.$. Hence $S^{\prime}$ contains a spherical neighborhood $S(p, \beta)(\beta>0)$ such that $S(q, \alpha) \cap S(p, \beta)=\phi$. Then there exists a positive integer $N$ such that $S(p, \beta)$ contains $p_{n}$ for every $n \geqq N$. At this time, $S(p, \beta)$ is simply covered by a system of asymptotes whose initial points do not belong to this neighborhood. This contradicts $K(\mathfrak{l}) \ni p_{n}(n=1,2, \ldots)$. Thus the theorem is proved.

Now, if an E-space is a 2-dimensional differentiable Finsler manifold we can then get a further conclusion. We shall show this in the next paragraph.

5. In this paragraph, our purpose is to prove the following theorem.

(5. 1) THEOREM. IIn an unbounded 2-dimensional Finsler manifold $\tilde{i}$ of class $C^{r}(r \geqq 4)$, if there exists only one coray to a ray $\mathfrak{l}$, then the set $K(\mathfrak{l})$ is empty.

Proof. It is clear that the property of domain invariance is, of course, satisfied in $\mathfrak{F}$. Hence the set $K(\mathfrak{l})$ is closed in $\mathfrak{v}$. Let $\mathfrak{A}$ be an asymptote to the ray $l$ and $a$ be its asymptotic conjugate point. We shall prove that if such a point $a$ exists, then we arrive at a contradiction.

If we take a sufficiently small positive number $\alpha$, then $S^{\prime}(a, \alpha)=\{x \mid a x$ $=\alpha\}$ is disjoint from all asymptotes which are straight lines. Let $S^{\prime}(a, \alpha)$ be such a neighborhood, and take on $\mathfrak{A}$ a point $p$ such that $\alpha<a p$. In the same way as in the proof of (4.3) we can see that the system of asymptotes containing a point of $S^{\prime}(a, \alpha)$ simply cover a neighborhood of the point $p$. Then such a neighborhood of $p$ contains a circular neighborhood $S^{\prime}(p, \beta)(\beta$ $>0)$ such that $S^{\prime}(a, \alpha) \cap S^{\prime}(p, \beta)=\phi$ and $S^{\prime}(p, \beta) \cap K(\mathfrak{l})=\phi$.

Consider in $S^{\prime}(p, \beta)$ a differentiable are $L$ through $p$ which does not 
intersect $\mathfrak{A}$ except at $\boldsymbol{p}$, and suppose that every asymptote having a common point with $L$ has no other common points. If the length of $L$ is sufficiently small, then every asymptote having a common point with $L$ has necessarily the asymptotic conjugate point. If this were not so, then we should have a sequence of asymptotes $\left\{\mathfrak{U}_{n}\right\}$ such that each $\mathfrak{A}_{n}$ is a straight line and intersects $L$ at a point $p_{n}$ whose sequence converges to the point $p$. It is easy to see that the sequence of asymptotes $\left\{\mathfrak{U}_{n}\right\}$ converges to $\mathfrak{A}$. Hence $\mathfrak{A}$ is a straight line, which contradicts $a \in K(\mathfrak{l})$.

The set $L^{\prime}$ of the initial points of all asymptotes which intersect $L$ is in one-to-one correspondence with $L$. Furthermore this correspondence is bicontinuous. Hence $L^{\prime}$ is an arc containing the point $a$.

By the point $p$, the $\operatorname{arc} L$ is divided into the two $\operatorname{arcs} L_{1}$ and $L_{2}$, let $L_{1}$ and $L_{2}$ intersect the boundary of $S^{\prime}(p, \beta)$ at points $p_{1}$ and $p_{2}$ respectively, where $\bar{\beta}$ is a suitable positive number smaller than $\beta$, and let $p_{1}^{\prime}$ and $p_{2}^{\prime}$ be respectively the image of $p_{1}$ and $p_{2}$ in the above correspondence. The arc $L^{\prime}$ clearly contains $p_{1}^{\prime}$ and $p_{2}^{\prime}$, and any one of these three points $p_{1}^{\prime}, p_{2}^{\prime}$, and $a$ does not coincide with the others. The boundary of a neighborhood $S^{\prime}(a$, $\bar{\alpha})\left(0<\bar{\alpha}<\min \left(\boldsymbol{a} \boldsymbol{p}_{1}^{\prime}, \boldsymbol{a} \boldsymbol{p}_{2}^{\prime}\right)\right.$ intersects $L^{\prime}$.

Let $\widetilde{p_{1}^{\prime}}$ and $\widetilde{p_{2}^{\prime}}$ be the points at which $L^{\prime}$ intersects the boundary of $S(a$, $\bar{\alpha})$ at first from $a$ and $\widetilde{L^{\prime}}$ be the subarc of $L^{\prime}$ whose and points are $\widetilde{p_{1}^{\prime}}$ and $\widetilde{p_{2}^{\prime}}$. Then $\widetilde{L^{\prime}}$ divides $S^{\prime}(a, \bar{\alpha})$ into two domains $D_{1}$ and $D_{2}$.

On the other hand, the set $K(\mathfrak{l})$ does contain all points of $S^{\prime}(a, \alpha)$. Hence there exists a point $x \bar{\in} K(\mathfrak{l})$ and a neighborhood $S^{\prime}\left(a, \overline{\alpha^{\prime}}\right)$ such that $S^{\prime}\left(x, \overline{\alpha^{\prime}}\right)$ $\subset S^{\prime}(a, \alpha) \cap(\widetilde{\mho}-K(\mathfrak{l}))$, since the set $K(\mathfrak{l})$ is closed.

If $q$ is a point of $\widetilde{L^{\prime}}-\widetilde{p_{1}^{\prime}}-\widetilde{p_{2}^{\prime}}$, then $S^{\prime}(q, \rho) \cap D_{i} \cap(\widetilde{\mho}-K(\mathfrak{l}) \neq \phi(i=1,2)$ for any positive number $\rho$. The asymptote $\mathfrak{x}$ from $q$ to $l$ is locally contained in one of the two domains $D_{1}$ and $D_{2}$. Let $\mathfrak{x}$ be locally contained in the domain $D_{1}$. In this case the domain $D_{2}$ does not locally contain $x$ and contains a sequence of points $\left\{\boldsymbol{q}_{n}\right\}\left(\boldsymbol{q}_{13} \in K(\mathfrak{l})\right.$ which converges to the point $q$. The coray $x_{n}$ from each $q_{n}$ to $\mathfrak{l}$ is locally contained in the domain $D_{2}$, and the sequence of corays $\left\{\mathfrak{x}_{n}\right\}$ converges to the asymptote $\mathfrak{x}$.

Let $r$ be a point on $\mathfrak{x}$ such that $q r=k$ and $T(q, r) \subset S^{\prime}(a, \alpha)$, where $k$ is a positive number, and let $\left\{r_{n}\right\}$ be a sequence of points such that each $r_{n}$ lies on $\mathfrak{x}_{n}$ and $\boldsymbol{q}_{n} \boldsymbol{r}_{n}=k$. Then $\left\{\boldsymbol{r}_{n}\right\}$ converges to the point $r$, and the sequence of segments $\left\{T\left(q_{i}, \boldsymbol{r}_{n}\right)\right\}$ uniformly converges to the segment $T(q, r)$. Hence there exists a positive integer $N$ such that $T\left(q_{n}, \boldsymbol{r}_{n}\right) \subset S^{\prime}(a, \bar{\alpha})$ for eve ev $^{-} n \geqq$ $N$. From this each $\mathfrak{x}_{n}(n \geqq N)$ intersects $\widetilde{L}^{\prime}$. But the corays $\mathfrak{x}_{n}$ are all disjoint from the set $K(\mathfrak{l})$. Thus we see that the asymptotic conjugate point $a$ does not exist. This proves the theorem.

The most interesting problem in differentiable cases is to investigate precisely the behavior of anasymptotes at an asymptotic conjugate point. This problem was completely solved in the case where the set of asymptotic 
conjugate points to a given ray contains an isolated point. In the later paper, we shall deal with such a problem on a Riemannian surface with non-positive Gaussian curvature which is homeomorphic to the sphere punctured at a finite number of points.

\section{REFERENCES}

[1] E. CARTAN, Leçon sur la géométrie des espaces de Riemann, Paris (1947).

[2] H. BusemanN, Local metric geometry, Trans. Amer. Math. Soc., Vol. 56(1944) 200-274.

[3] H. BusEmanN, Spaces with non-positive curvature, Acta, Math., Vol. 80 (1948), 259310.

[4] F. Hausdorff, Mengenlehre, dritte Aufgabe, Berlin (1935).

[5] P. HARTMANN and A. WintneR, Gaussian curvature and local embedding, Amer. Journ. Math., Vol. 73(1953), 876-884.

KUMAMOTO UNIVERSITY, 